\section{POPULATION ECOLOGY}

\section{Genomic futurology}

Nature 573, 126-129 (2019).

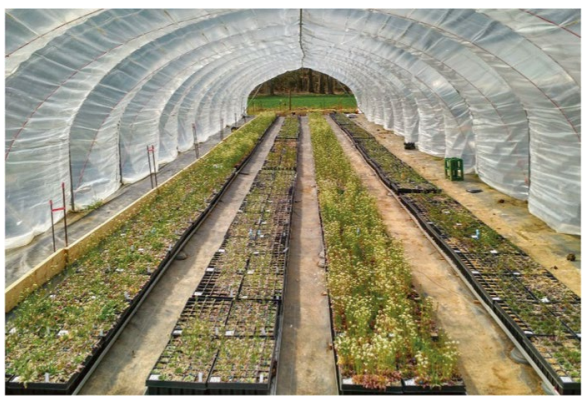

Credit: Springer Nature Ltd

When the possible effects of climate change on biodiversity are discussed, the conversation usually revolves around which species will change their range or become extinct rather than changes in population structures within individual species. But this is exactly the question that Moises Exposito-Alonso of the Max Planck Institute for Developmental Biology in Tübingen, Germany, and colleagues have addressed using the plant biologist's favourite model organism, Arabidopsis thaliana.

The researchers investigated the growth of 517 different populations of Arabidopsis originally collected from across the species' range from California through Europe and central Asia to Japan. They planted seeds from these ecotypes in two locations: Tübingen, which is in the middle of the plants natural range and so in the 'Goldilocks zone' for growth, "not too hot, and not too cold"; and Madrid, Spain, which presents a hotter and more hostile environment. At each site, seeds from every ecotype were subjected to combinations of high and low water availability, and cramped as opposed to isolated conditions. In Germany, at least some plants of all ecotypes grew successfully under all conditions, but the more extreme climate of Spain saw only 193 of the 517 ecotypes survive in the most stressful condition of low water and crowding.

The unprecedented size of the data set produced by these logistically challenging experiments enabled the researchers to map fitness onto 1,353,386 single nucleotide polymorphisms - which vary between the ecotypes - under each of the conditions. This genomic map was then used to make a geographical map predicting how the genetics of the species are likely to be affected by anticipated changes in climate. The greatest changes will be seen in the transition zone between the Mediterranean and temperate regions, with many alleles coming under increasingly negative selective pressure resulting in the predicted disappearance of many ecotypes and the diversity of alleles they carry, even if Arabidopsis as a species continues to flourish.

For a creature as abundant and widely distributed as Arabidopsis, such changes are simply natural selection in action. However, by predicting the precise effects of environmental change on defined local populations, these researchers' approach could greatly aid the design of conservation strategies for more vulnerable plants and animals.

Chris Surridge

Published online: 7 October 2019

https://doi.org/10.1038/s41477-019-0532-7 